\title{
MAPEAMENTO ESPAÇO - TEMPORAL DE UMA DÉCADA DE INCIDÊNCIA DE ASMA EM CRIANÇAS EM MUNICÍPIOS PAULISTAS
}

\author{
SPATIAL-TEMPORAL MAPPING OF ONE DECADE OF ASTHMA INCIDENCE FOR CHILDREN IN \\ THE SÃO PAULO MUNICIPALITIES
}

\author{
Milton Mendes Cattini ${ }^{\mathrm{A}}$ \\ Renata Calciolari Rossi Silva ${ }^{\mathrm{A}}$ \\ Marcus Vinicius Pimenta ${ }^{\text {A }}$ \\ (D) Rogério Giuffrida ${ }^{\mathrm{A}}$ \\ Rejane Ennes Cicerelli ${ }^{\text {B }}$ \\ (iD) Lucas Prado Osco ${ }^{\mathrm{A}}$ \\ (iD) José Marcato Junior ${ }^{\mathrm{C}}$ \\ Bruna Maria Casachi Bernardes de Melo Carapeba ${ }^{\mathbf{A}}$ \\ (iD) Ana Paula Marques Ramos ${ }^{\mathbf{A}}$ \\ ${ }^{\text {A }}$ Universidade do Oeste Paulista (Unoeste), Presidente Prudente, SP, Brasil \\ ${ }^{\text {B } U n i v e r s i d a d e ~ d e ~ B r a s i ́ l i a ~(U n B), ~ B r a s i ́ l i a, ~ D F, ~ B r a s i l ~}$ \\ ${ }^{\mathrm{C}}$ Universidade Federal do Mato Grosso do Sul (UFMS), Pioneiros, MS, Brasil
}

\author{
Recebido em: 20/07/2021 | 08/09/2021 DOI: 10.12957/tamoios.2021.61191 \\ Correspondência para: Milton Mendes Cattini (miltoncattini@gmail.com)
}

\section{Resumo}

A asma é um problema de saúde pública que acomete, sobretudo, crianças. Esse trabalho mapeia uma década da taxa de incidência de asma em crianças (1 a 14 anos) nos municípios da $11^{\mathrm{a}}$ Rede Regional de Atenção à Saúde (RRAS-11) do estado de São Paulo. Um total de 45 municípios, entre 2008 e 2018, foi estudado com os dados do DATASUS. A taxa de incidência representa casos a cada 1.000 crianças. Não há dependência espacial (Índice de Moran $=0,131 ; Z=1,200 ; p$-valor $=0,230$ ) entre os municípios e a doença nessa faixa etária. Alguns locais são mais acometidos pela asma que outros, caracterizando uma distribuição heterogênea de asma na RRAS-11 no período analisado. Há uma associação entre taxa de asma e sazonalidade climática, sendo os períodos de inverno e outono os mais críticos para a ocorrência da doença. O estudo mostra uma tendência de diminuição nos casos de asma em crianças de 2008 a 2018 na RRAS-11. Esses achados contribuem para o direcionamento de futuras ações públicas de saúde aos municípios mais acometidos pela enfermidade. A metodologia usada pode ser aplicada em outras regiões de saúde para caracterização desse tipo de doença na faixa etária de interesse.
\end{abstract}

Palavras-chave: doença respiratória; análise espacial; geoprocessamento; mapa temático.

\begin{abstract}
Asthma is a public health problem that affects mainly children. Herein we map a decade of the incidence rate of asthma in children (1 to 14 years old) in the municipalities of the 11th Regional Health Care Network (RRAS-11) in the state of São Paulo. A total of 45 municipalities between 2008 and 2018 were studied using the DATASUS dataset. The incidence rate is cases per 1,000 children. There was no spatial dependence (Moran's Index $=0.131 ; Z=1.200 ; p$-value $=0.230$ ) between municipalities and the disease in this age group. Some sites are more affected by asthma than others, characterizing a heterogeneous distribution of asthma in the RRAS-11 in the period analyzed. There is an association between the rate of asthma and climatic seasonality, with the winter and autumn periods being the most critical for the occurrence of the disease. The study shows a decreasing trend in asthma cases in children from 2008 to 2018 in the RRAS-11. These findings contribute to directing future public health actions to the municipalities most affected by the disease. The methodology used can be applied in other health regions to characterize this type of disease in the age group of interest.
\end{abstract}

Keywords: respiratory disease; spatial analysis; geoprocessing; thematic maps. 


\section{INTRODUÇÃO}

A asma é uma doença inflamatória crônica de vias respiratórias caracterizada por hiperresponsividade brônquica, sendo manifestada por obstrução do fluxo aéreo intrapulmonar generalizado e com possíveis variações. A reversibilidade dessa doença pode ser espontânea ou por meio de tratamento medicamentoso, com dependência estrita do seguimento médico e cuidados rigorosos com mecanismos desencadeantes. Clinicamente, a asma pode se apresentar em diferentes formas, sendo decorrente de uma relação entre três elementos fundamentais: fator genético, comportamento celular alterado e exposição a fatores ambientais e/ou físico-biológico (FRANCO et al., 2006).

Considerada um problema de saúde pública, a manifestação da asma pode ser caracterizada por sintomas ocasionais e de baixa magnitude, que às vezes estão associados à poluição atmosférica, infecções das vias aéreas ou exposição aos componentes alérgenos (CAMPOS, 2015). Os desencadeantes da reação de hipersensibilidade podem ser específicos alérgenos inaláveis, substâncias derivadas de ácaros, animais domésticos, baratas, pólen e fungos do ar; ou agentes inespecíficos, como infecções virais, tabagismo - ativo ou passivo, substâncias concomitantes à poluição e até medicamentos (SILVA e DIAS, 2013). A exposição ambiental é representada ao correspondente de partículas inaladas, exposições ocupacionais e irritantes do trato respiratório, das quais são de difícil eliminação, em que muitas vezes estão inseridos os doentes asmáticos, tornando uma tarefa complicada de prevenção e exclusão total. O controle ambiental e dos fatores agravantes da doença são de importância fundamental na minimização dos sintomas (MANNINO et al., 2002; BARNETT et al., 2005).

Todas as faixas etárias são acometidas pela asma, com dados que demonstram alta prevalência, morbidade e mortalidade no mundo (EDER et al., 2006; GINA, 2016). A prevalência da asma pode ser considerada alta em diversos países, o que traduz em associação íntima e em uma relação de causa e consequência, que se manifesta e representa impactos negativos à saúde pública mundial (BEASLEY, 1998; LAI et al., 2009). As populações com maior prevalência de asma estão em países de língua inglesa e na América Latina (NETO et al., 2012). A enfermidade apresenta uma evolução espaço temporal associada a fatores de risco preponderantes para o desencadeamento dos sintomas, associada às intercorrências biológicas, econômicas, limitações aos serviços de saúde, falta de medicamentos e fatores atmosféricos.

No aspecto sintomático, em 2015, verificou-se em crianças em idade escolar que 17,92\% relataram possuir um episódio da doença em algum período da vida (SILVA et al., 2018). Ademais, uma porcentagem de $23,52 \%$ referiu sintomas relacionados ao chiado no peito (SILVA et al., 2018). De acordo com a Pesquisa Nacional de Saúde do Escolar, houve um aumento da prevalência no Brasil de 3,6\% em 2015 com relação a 2012, principalmente na região sul do país (MARQUES et al., 2019). Estimativas contidas no Relatório Global de asma de 2018 evidenciaram que mais de 330 milhões de pessoas no mundo são afetadas pela 
asma, com uma expectativa de aumento para o futuro. No Brasil, o número aproximado é de 20 milhões de pessoas, sendo a maior parte na faixa etária infantil (RONCADA et al., 2018).

A poluição atmosférica é um fator de importância para o desenvolvimento do quadro sintomático, principalmente em crianças e idosos, que são as faixas etárias mais vulneráveis à enfermidade, considerando fatores como o meio ambiente inserido e as partículas geradas pela queima da biomassa (CANÇADO et al., 2006). As crianças são mais expostas aos poluentes atmosféricos por realizarem ciclos respiratórios de duração mais curta, maiores níveis de atividade física e exposição mais frequente ao ar livre quando comparado aos adultos (AMÂNCIO et al., 2012; CHAVES et al., 2017). As doenças respiratórias na infância podem ser prevenidas com diversas medidas de caráter básico como, por exemplo, melhoria no fator nutricional, evitar a exposição à fumaça do tabaco e alérgenos, além de imunização adequada e tratamento efetivo de outras comorbidades existentes (CHAVES et al., 2017). Deve-se também priorizar educação e formação profissional para um manejo correto e uma conduta adequada, desde à atenção básica na rede de promoção e prevenção, até o atendimento de urgência/emergência nos casos de crises (ZAR et al., 2014).

A falta de tratamento e não adesão ao esquema farmacológico proposto ocasionam períodos de crises e aumentam os riscos de complicações respiratórias. Isso pode levar, muitas vezes, a quadros emergenciais e internações hospitalares, acarretando maior uso do dinheiro público e piora na qualidade de vida do paciente (RONCADA et al., 2018). Isso reforça a importância de estratégias que reduzam o impacto dessa doença na faixa etária de maior prevalência do quadro asmático.

O impacto que a asma ocasiona na economia do país deve ser considerado para os cofres públicos. O Ministério da Saúde e o Instituto Brasileiro de Geografia e Estatística (IBGE), por meio da Pesquisa Nacional de Saúde (PNS), publicada em 2014, divulgou que, em 2013, a asma atingiu 4,4\% de brasileiros com 18 anos ou mais, sendo as mulheres as mais acometidas $(5,1 \%)$ em relação aos homens $(3,6 \%)$. Somente em 2014, no período de janeiro a novembro, foram 105,5 mil internações pela doença originando um custo de $\mathrm{R}$ \$ 57,2 milhões para a rede pública de saúde, segundo dados do Sistema de Informações Hospitalares (SIH). Contudo, não se pode avaliar o custo apenas com a enfermidade propriamente dita, deve-se considerar os aspectos relacionados aos fatores econômicos, como abstenção ao trabalho em períodos de crise - licenças de saúde, baixa produtividade por comprometimento respiratório, doenças associadas, transportes ao local de atendimento e até pagamento de auxílios pelo governo (COSTA et al., 2018). Um estudo (RODRIGUES et al., 2015) realizou a projeção de internações hospitalares e óbitos no sistema público de saúde do Estado de São Paulo, tendo como causa à poluição atmosférica, no período de 2011 a 2030. A estimativa demonstra mais de 246 mil óbitos e 918 mil internações por enfermidades cardiovasculares, de via aérea e neoplasias. Em relação às doenças respiratórias em crianças, esse mesmo estudo aponta ocorrer acima de 138 mil internações com um gasto superando 134 milhões de reais. 
Diferentemente de outros países em desenvolvimento, nos quais grandes bancos de dados nacionais são incomuns, o Brasil possui o Sistema de Informações Hospitalares do SUS (SIH/SUS). Um banco de dados de livre acesso do Ministério da Saúde registra indicadores de saúde, como por exemplo, dados de morbidade relacionados a doenças respiratórias. Essas informações são essenciais para melhorar e intensificar as políticas de saúde para doenças de maior prevalência, como a asma, e em grande escala geográfica, capaz de atingir números que representam escalas nacionais, estaduais ou regionais/municipais. Embora haja estudos sobre impacto da asma no Brasil (VALENÇA et al., 2006; SILVA et al., 2009; RODRIGUES et al., 2010; WEHRMEISTER et al., 2012; FILHO et al., 2013; RODRIGUES et al., 2013; CARDOSO et al., 2017; SOUZA et al., 2017; FERNANDES et al., 2018; RAMOS et al., 2019), assim como de outras doenças, tais como a COVID-19 (CARDOSO et al. 2020; DO VALE, 2020), até o momento, nenhum trabalho aborda a questão da distribuição multitemporal da incidência de asma em crianças na região Oeste do estado de São Paulo; uma área caracterizada por forte desenvolvimento do setor sucroalcooleiro no Brasil (RAMOS et al., 2019). Há apenas dois estudos na temática de doenças respiratórias nessa região, o de Vioto et al. (2017) e Ramos et al. (2019). No primeiro, fez-se o mapeamento da taxa de asma em idosos em 32 municípios no período de 2008 a 2015. Todavia, Vioto et al. (2017) consideram a notificação de casos por local de internação, e, em estudos epidemiológicos, a notificação deveria ocorrer por local de residência (MARINHO et al., 2019). No estudo de Ramos et al. (2019), apenas um município (Presidente Prudente) da região supracitada é considerado e os autores avaliam a associação entre internações hospitalares por doenças respiratórias em geral, emissões de poluentes atmosféricos e variações meteorológicas.

Diante do exposto, esse trabalho pretende preencher essa lacuna, e visa caracterizar a distribuição espacial, ao longo de mais de uma década (2008 a 2018), da taxa de incidência de asma em indivíduos de 1 a 14 anos nos municípios da $11^{\text {a }}$ Rede Regional de Atenção à Saúde (RRAS-11) do estado de São Paulo, formada por 45 municípios. A principal contribuição desta pesquisa é responder questões de orientação de políticas públicas de saúde na região da RRAS-11 do estado de São Paulo. A saber: "Qual a taxa de incidência da asma em crianças na última década na RRAS-11?"; "Existe dependência espacial entre os municípios dessa região em relação a taxa de asma em indivíduos dessa faixa etária de 2008 a 2018?"; "Há associação entre a sazonalidade climática e a taxa de incidência de asma nesses municípios no período estudado?"; "Há correlação desses casos com os focos de queimada na região?"; "Qual a tendência de distribuição da taxa de asma em crianças de 1 a 14 anos de 2008 até 2018 na RRAS-11?". Para vencer tais desafios, o método empregado consiste na utilização de técnicas de representação quantitativa da cartografia temática, como elaboração de mapas coropléticos para demostrar a distribuição espacial da taxa de asma por município no decorrer dos anos, e de técnicas de análise espacial, como o Índice Global de Moran, e a construção do LISA (Índice Local de Associação Espacial) Map, para evidenciar associação espacial entre a ocorrência da doença nos municípios. As demais seções deste trabalho estão organizadas da 
seguinte forma: na seção 2 se apresenta os materiais e método; os resultados e discussões encontram-se na seção 3, e a quarta seção conclui a pesquisa sobre a taxa de asma em crianças ao decorrer de mais de dez anos nos 45 municípios da RRAS- 11.

\section{METODOLOGIA}

Trata-se de um estudo descritivo da distribuição da taxa de asma em crianças de 1 a 14 anos nos municípios da RRAS-11 no período de 2008 a 2018. Essa área geográfica (Figura 1) está localizada à sudeste do Brasil, na região Oeste do estado de São Paulo, com extensão territorial de aproximadamente $22.334 \mathrm{mil} \mathrm{km²}$. A população é estimada em 722 mil habitantes, dos quais $90 \%$ residem em área urbana conforme o último Censo Demográfico (IBGE, 2010). A RRAS-11 abrange seis regiões de saúde: Alta Paulista, Alta Sorocabana, Alto Capivari, Extremo Oeste Paulista e Pontal Paranapanema do Estado de São Paulo.

Para o mapeamento da taxa de incidência nos municípios da RRAS-11, o método se constitui de: (i) levantamento do número de casos de asma entre indivíduos de 1 a 14 anos por município da RRAS-11 de 2008 a 2018; (ii) levantamento do número de indivíduos nessa faixa etária por município no censo demográfico de 2010 do IBGE; (iii) determinação da taxa de incidência de asma para o grupo de indivíduos de interesse por município entre 2008 e 2018; (iv) espacialização da taxa de incidência de asma, e de variáveis como pontos de queimada por município, utilizando técnicas da Cartografia Temática; (v) determinação do índice Global de Moran e construção do LISA MAP para estudo da dependência espacial entre os municípios em relação a taxa de asma em indivíduos dessa faixa etária de 2008 a 2018. Essas etapas são detalhadas a seguir.

Figura 1 - Localização dos municípios da RRAS-11 no Estado de São Paulo 


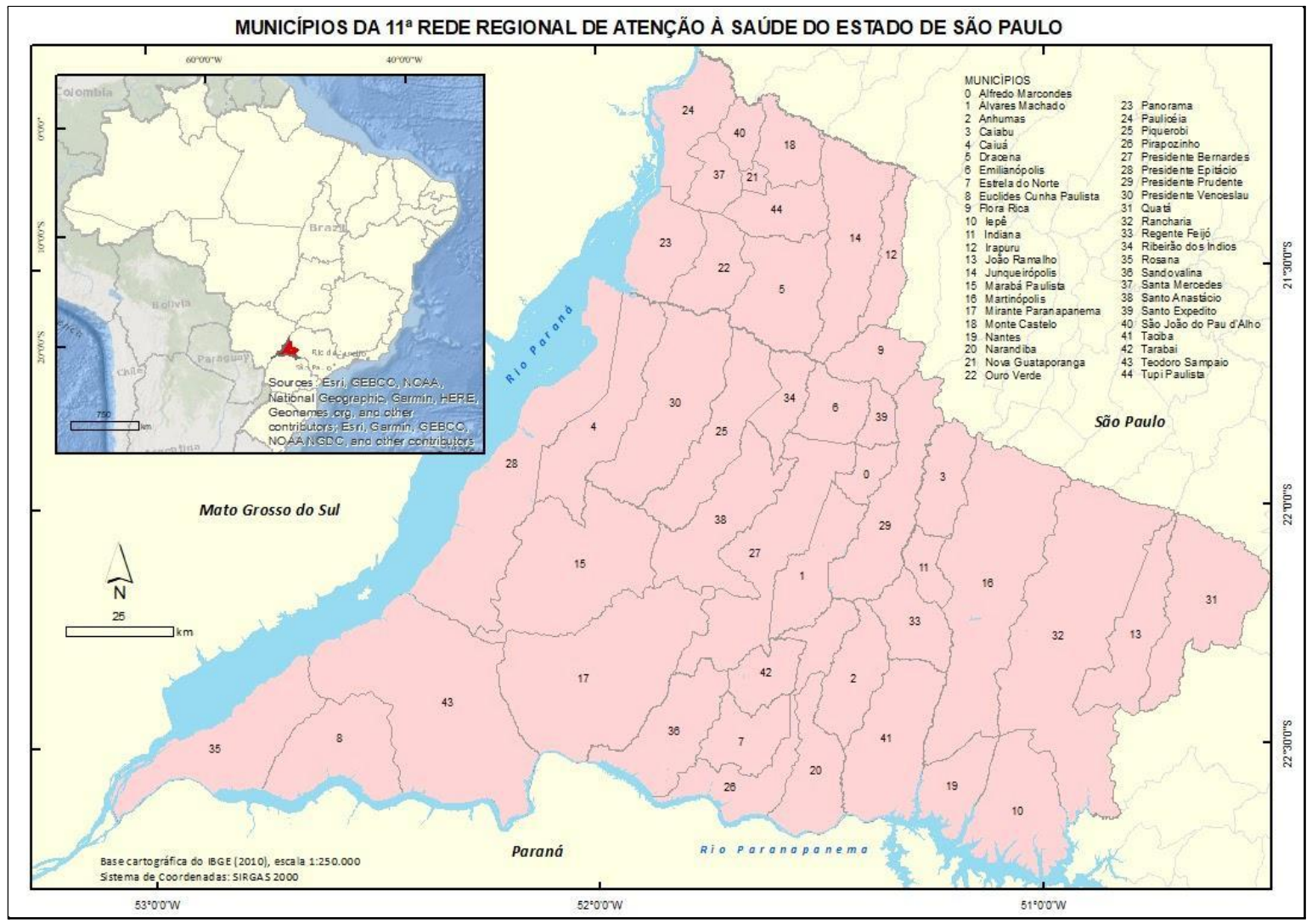

Fonte: Autores.

\section{Levantamento dos dados e cálculo da taxa de incidência}

A base de dados utilizada é formada por casos notificados de asma em crianças (1 a 14 anos) nos 45 municípios da RRAS-11 entre 2008 e 2018. A base cartográfica com o limite dos municípios foi obtida no banco de dados geográfico do IBGE, na escala 1:250.000, ano de 2018. A manipulação dessa base para a produção dos mapas de incidência de asma foi realizada no software de SIG da ESRI, o ArcGIS 10.4.

Os dados de asma em crianças foram acessados no banco de dados DATASUS (http://tabnet.datasus.gov.br/) e a consulta realizada por estado. A forma de agrupamento para a apresentação dos dados foi a seguinte: seleção dos casos por município de residência, período de mês a mês entre 2008 até 2018, e somente AIH (Autorização de Internação Hospitalar) aprovada. Os dados referentes ao número de crianças por município foram obtidos no censo de 2010. A amostra do presente estudo é constituída de 138.842 crianças (1 a 14 anos), representando aproximadamente $19 \%$ da população da RRAS- 11 . O período de 2008 a 2018 foi definido com base na disponibilidade dos registros de asma no DATASUS, caracterizando mais de uma década de distribuição da doença. 
A região da RRAS-11 tem clima tropical, com a presença de um período seco (estação do inverno) sob influência predominante dos sistemas polares e um período chuvoso (estação do verão) sob influência dos sistemas tropicais (MONTEIRO, 2000). A precipitação anual média varia entre 1200 e $1500 \mathrm{~mm}$, enquanto a temperatura média anual permanece acima de $22^{\circ} \mathrm{C}$ (BOIN, 2000). Neste trabalho, a sazonalidade climática foi caracterizada por quatro períodos: verão (meses de dezembro, janeiro e fevereiro), outono (meses de março, abril e maio), inverno (meses de junho, julho e agosto) e; primavera (meses de setembro, outubro e novembro).

O cálculo da taxa de incidência, para cada um dos 45 municípios da RRAS-11, ocorreu dividindo o número absoluto de casos de asma em crianças no município e a população de crianças nesse mesmo município multiplicado pelo fator 1.000. Isso permitiu representar a taxa de incidência a cada 1.000 indivíduos. Esse valor é uma métrica indicada quando o denominador e numerador não são muito discrepantes entre si (DENT et al., 2009), o que se verificou na amostra de dados desse estudo no período 2008 a 2018.

\section{Mapeamento dos casos de asma e dos focos de queimadas na RRAS-11}

Para analisar a distribuição espacial da taxa de asma em crianças na RRAS-11 de 2008 a 2018, produzimos mapas quantitativos do tipo coroplético. Esses mesmos mapas foram construídos em função da variação da estação do ano (inverno, outono, verão e primavera). Obtivemos com isso a taxa média de incidência de asma entre 2008 e 2018, bem como a taxa média de incidência de asma por período sazonal climático em mais de uma década (2008 a 2018) na RRAS-11. Um mapa coroplético representa a quantidade relativa de um fenômeno por unidade de enumeração (DENT et al. 2009). Essa quantificação do fenômeno por unidade de enumeração é expressa no mapa por meio de um esquema de cores que denote uma ordem visual entre as classes de dados (KRYGIER e WOOD, 2005). Assim, cada cor apresentada na legenda simboliza um intervalo de classe de valores no mapa. $\mathrm{Na}$ construção dos mapas coropléticos, adotou-se a abordagem metodológica apresentada em Ramos et al. (2016), ao que se refere à definição do número de classes, método de agrupamento dos dados e construção dos mapas. Foram consideradas cinco classes de valores para representar a taxa de asma em crianças, o método de classificação foi o de Quebras Naturais (DENT et al., 2009), e os princípios da comunicação cartográficas aplicados na construção deles.

Os dados referentes aos focos de queimadas foram obtidos no banco de dados on-line do Instituto Nacional de Pesquisas Espaciais (INPE) ano a ano de 2008 até 2018. Esses dados foram representados no mapa coroplético de incidência de asma utilizando a geometria de pontos, para permitir uma análise visual entre os locais de onde ocorrem queimadas e a taxa de asma no município correspondente. Considerando que $90 \%$ da população reside nos centros urbanos foi realizada uma análise a partir dos focos de queimadas localizados em um raio de 10 quilômetros. 


\section{RESULTADOS E DISCUSSÃO}

No período de janeiro de 2008 a dezembro de 2018, o DATASUS contabilizou um total de 1.018 casos de asma em crianças de 1 a 14 anos na RRAS-11 do estado de São Paulo. O maior número de casos, 192, foi registrado em 2008, e o ano de 2016 apresentou o menor número com 42 casos (Figura 2). Pelo teste paramétrico t, de uma amostra simples, confirmamos $(\mathrm{t}=132,58$; $\mathrm{p}$-valor $<0,0001)$ ao nível de confiabilidade de $95 \%$, que alguns anos apresentaram número de casos de asma em crianças mais acentuado comparado com outros anos. Os valores absolutos dos casos de asma em crianças apresentaram distribuição de probabilidade normal, pelo teste de normalidade de Shapiro-Wilk (Valor do teste $=0,889$; $\mathrm{p}$-valor $=0,137)$.

Houve uma tendência decrescente $\left(\mathrm{r}^{2}=0,692\right)$ no número de casos de asma em crianças na RRAS-11 na última década. Até o ano de 2012, o número de casos reduziu progressivamente, apresentando uma oscilação entre os anos de 2013 até 2018. Apesar da falta de infraestrutura, e da precariedade do sistema público de saúde, no decorrer dos anos, ocorreram melhoras em fatores relacionados à campanha da atenção primária - Estratégia de Saúde da Família (AMARAL et al., 2012). Houve, também, uma evolução das políticas públicas e planos de controle para a asma. Diversos foram os pontos estabelecidos, desde capacitação e educação permanente de profissionais da saúde, aperfeiçoamento das coberturas, repasses de verbas, criação aprimoradas de diretriz pelas Sociedades Médicas de Pneumologia e Tisiologia, modelos assistências e até a distribuição gratuita de medicamentos para tratamento e manejo da enfermidade (AMARAL et al., 2012).

Um estudo descritivo (CARDOSO et al., 2017) sobre a distribuição dos casos de asma entre 2008 e 2013 para diversas regiões do Brasil revela uma redução de 10\% no número de óbitos pela doença, além da diminuição em 36\% de sua hospitalização. Os resultados de nosso trabalho (Figura 2) demonstra, no contexto epidemiológico, achados similares, uma vez que constatamos uma queda do número de casos confirmados de asma em relação ao período estabelecido (2008 a 2018).

A Figuras 3 apresenta a distribuição espacial da taxa de incidência de asma em crianças nos 45 municípios da RRAS-11 no período de 2008 a 2018. Identificamos uma distribuição heterogênea da incidência de asma em crianças nesses municípios; ou seja, em algumas regiões da RRAS-11 as crianças de 1 a 14 anos são mais acometidas por essa doença respiratória que em outras. Verificamos, ainda, que em apenas 11\% (do total de 45) dos municípios (Santo Anastácio, Presidente Venceslau, Ribeirão dos Índios, Teodoro Sampaio e Iepê) da RRAS-11 estão concentrados mais de 30\% (do total de 1.018) dos casos de asma em crianças. Nessas regiões, a taxa de incidência atingiu valores de até 22,34 a cada 1.000 crianças entre 2008 e 2018. Em contrapartida, outras cidades (Figura 3) possuem taxas de 
incidência em níveis mais baixos, como em Pirapozinho, Anhumas, Santo Expedito, Ouro Verde, Panorama, Santa Mercedes e Paulicéia.

De acordo com o Sistema de Estimativa de Emissão e Remoções de Gases do Efeito Estufa - SEEG, os municípios podem ser classificados de acordo com a projeção da quantidade de gases residuais eliminados nos últimos anos. No ano de 2016, todos os municípios com as maiores incidência de asma (Figura 3), ocupavam as seguintes posições de acordo com o SEEG - Teodoro Sampaio em $122^{\circ}$, Iepê em $373^{\circ}$, Santo Anastácio em $185^{\circ}$, Ribeirão dos Índios em $449^{\circ}$ e Presidente Venceslau em $111^{\circ}$. Sem embargo, quando se analisa do ponto de vista econômico, populacional, de saúde pública, e de desenvolvimento, devemos levar em consideração o município de Presidente Prudente, que no ranking descrito acima, ocupa a posição de $32^{\circ}$ para eliminação de gases. Sendo assim, nota-se que possui umas das piores classificações em comparação com todo o Oeste do Estado de São de Paulo, tendo uma emissão total alocada entre os anos de 2008 e 2016 que ultrapassa a média de 629 mil tCO2e, com participação responsável principalmente em energia, resíduos e agropecuária.

Figura 2 - Número absoluto de casos de asma em crianças na RRAS-11 de 2008 a 2018. A linha contínua, em azul, representa a distribuição de casos ao longo do período de 2008 a 2018. A linha reta tracejada, em azul, representa a reta de regressão linear para esse mesmo período de total de casos de asma em crianças

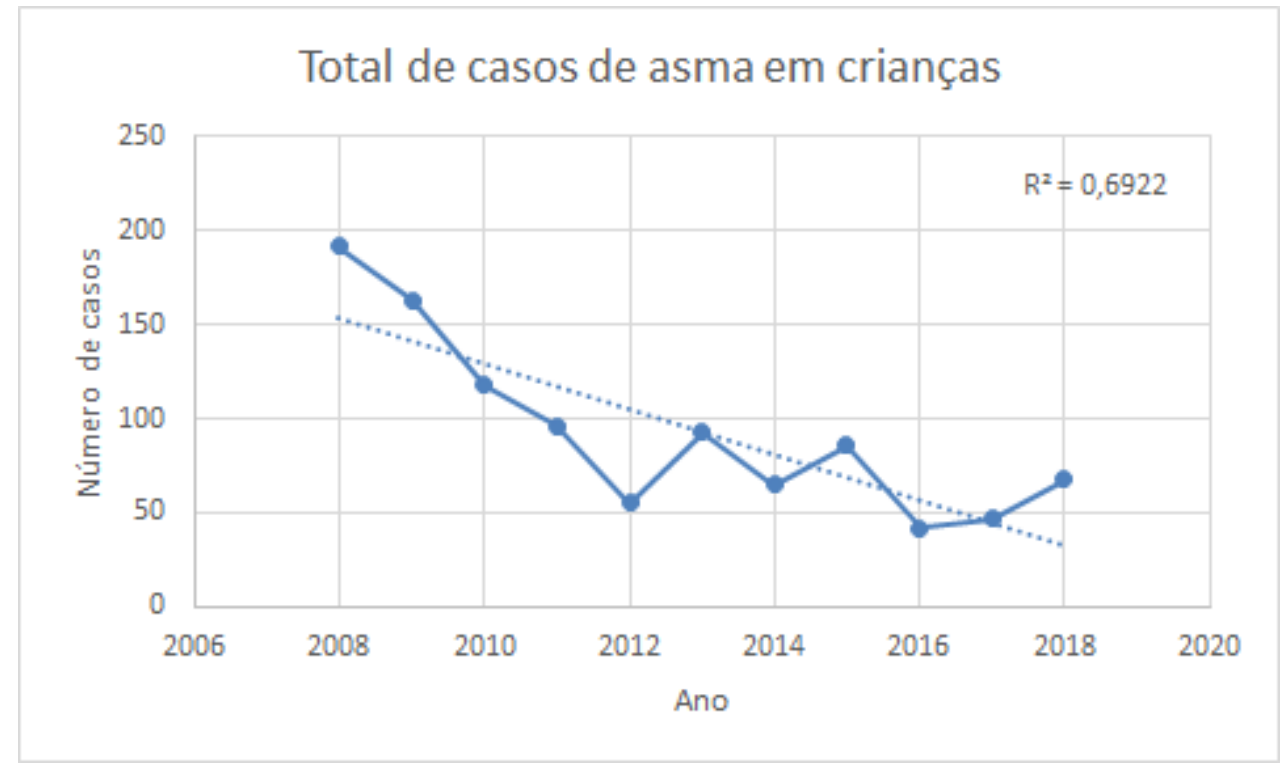

Fonte: Autores.

Observando os municípios representados na Figura 3, pode-se inferir que a formação de materiais poluentes não possui relação com a alta incidência de asma em Presidente 
Prudente, por exemplo, que apresenta uma incidência baixa e ocupa uma das piores posições $\left(32^{\circ}\right)$ da região na estimativa, segundo o SEEG Brasil. Resultado similar foi encontrado em um estudo (RODRIGUES et al., 2013) espaço-temporal no Estado de Rondônia, entre 2001 e 2010, que relatou a não associação da qualidade do ar por queimadas e internações por doenças respiratórias em crianças. Quando analisado o principal município da região - Porto Velho, os autores observaram que nos últimos 10 anos houve aumento de focos da poluição, com diminuição da taxa de internação para enfermidades de via aérea, equivalente à associação encontrada em nosso estudo. Em relação ao município de Teodoro Sampaio, a alta taxa de asma (Figura 3), pode estar associada a avenida principal da cidade ser a rodovia de acesso a várias cidades do Paraná e Mato Grosso do Sul.

O Índice Global de Moran, e o LISA Map (Figura 4) correspondente, mostrou uma associação espacial positiva, mas com valor muito baixo e próximo de zero (valor do índice 0,131; $Z=1,200 ; p$-valor $=0,230)$, refutando a hipótese de autocorrelação espacial. O valor inferido evidencia que os casos de asma de um município são poucos correlacionados com o valor médio de seus municípios vizinhos. O índice Global de Moran é uma técnica estatística que mede a autocorrelação espacial com base em localizações de feições (ex.: municípios) e valores de atributos (ex.: taxa de asma) por meio da estatística I de Moran. Trata-se de um indicador global de autocorrelação espacial, fornecendo um único valor como medida da associação espacial para todo o conjunto de dados (45 municípios neste caso de estudo). Por outro lado, o LISA MAP permite uma análise de padrões em escala de maior detalhe, produzindo um valor específico para cada objeto, permitindo assim, a identificação de agrupamentos de objetos (municípios) com valores de atributos semelhantes (taxas de asma). Para maiores detalhes sobre o LISA MAP e índice global de Moran, consultar Anselin (1995). Tais técnicas são utilizadas com recorrência em estudos (Braz et al. 2014; Thompson et al. 2018; Ren et al. 2020) que envolvem variáveis epidemiológicas, como a tratada aqui.

$\mathrm{Na}$ distribuição das taxas de incidência de asma em crianças por período sazonal (Figura 5), o outono e o inverno são os mais críticos para a ocorrência dessa doença. Nas estações de verão e primavera, as maiores taxas médias foram registradas apenas em cinco e três regiões, respectivamente. Ao passo que esse número cresce para 12 e 20, nas estações de inverno e outono, respectivamente. $\mathrm{Na}$ estação do outono aproximadamente $44 \%$ dos municípios da RRAS-11 apresentaram as maiores taxas de incidência de asma em crianças no período de 11 anos (2008 a 2018). Dentre os mecanismos relacionados à enfermidade, sabe-se que fatores associados aos agentes físico-biológicos, e até mesmo ambientais, podem agravar, exacerbar e desencadear a enfermidade descrita (FRANCO et al., 2006). Em um estudo realizado por Ezequiel et al. (2007), composto por mais de 25 mil atendimentos em pacientes com crise asmática, cuja faixa etária em mais de $80 \%$ era menor de 12 anos, evidenciou-se que o predomínio dos atendimentos em serviço de urgência/emergência ocorreu nos meses de maio, junho e julho. Isso demonstra a relação íntima entre a geografia espaço-temporal e a fisiopatologia da asma. 
Figura 3 - Distribuição espacial da taxa de asma em crianças na RRAS-11 de 2008 a 2018.

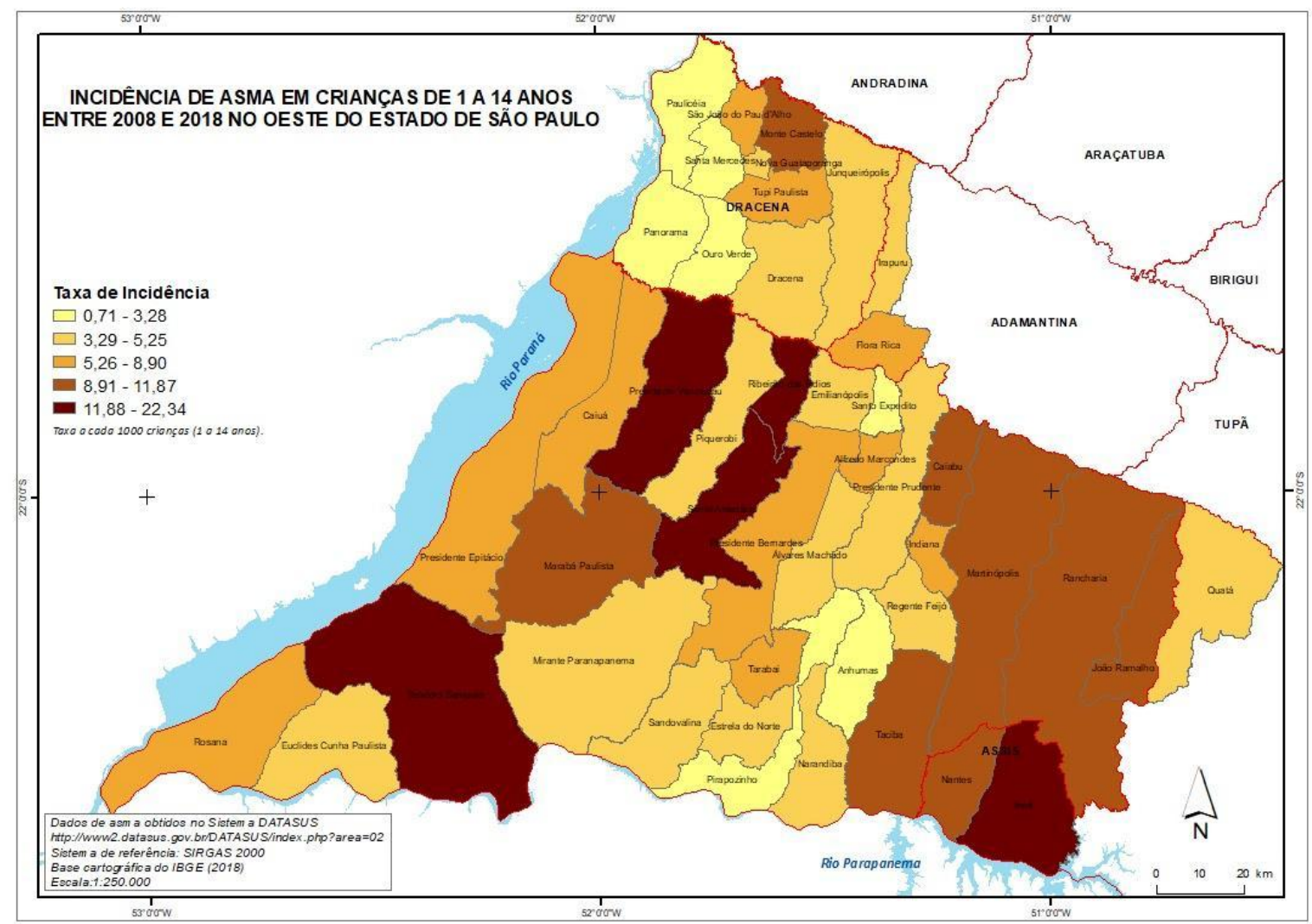

Fonte: Autores.

Em relação a maior cidade em número populacional do Oeste Paulista, Presidente Prudente se enquadra com baixa incidência (Figura 3), não apontando associação com fatores climáticos - estações do ano (Figura 5). Não obstante, quanto a sua classificação em nível socioeconômico, ocupa um dos melhores níveis do país. Esse quadro é baseado no Índice de Desenvolvimento Humano (IDH) de 2010, em que foram analisados fatores como educação, longevidade e renda. O município, segundo dados do IBGE, possui um valor de 0,806 - o que coloca o território na posição de $25^{\mathrm{a}}$ entre os municípios brasileiros, sendo, por conseguinte, considerado um valor numérico muito alto. Um grupo em situação economicamente 
desfavorecida apresenta relação maior com asma diagnosticada, períodos de crise, sibilos, chiados no peito, tosse, desconforto em sono e alteração na fala, dentre outros sintomas relacionados (FELIZOLA et al., 2005; RODRIGUES e PITREZ, 2012). Nosso estudo mostra que a cidade de Presidente Prudente dispõe de razões que, entre outros aspectos, são considerados protetores para a incidência da enfermidade asmática. 
Figura 4 - LISA Map para a distribuição espacial da taxa de asma em crianças na RRAS-11 de 2008 a 2018 por período sazonal.

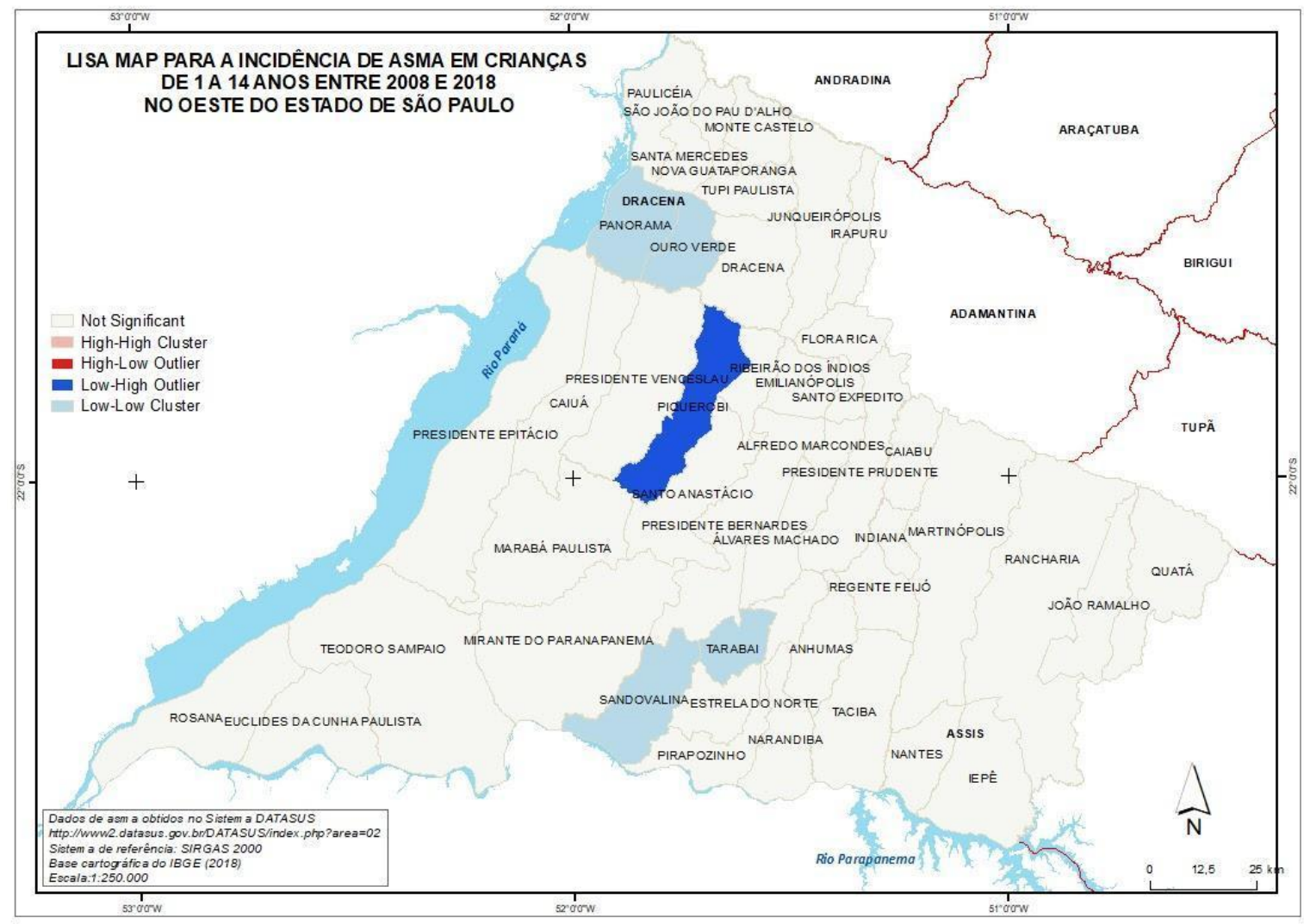

Fonte: Autores.

O mapa da Figura 6 apresenta a distribuição espacial de um total de 1.804 focos de queimadas no ano de 2008 (ano de maior número de casos de asma na área em estudo) nos 45 municípios da RRAS-11, bem como a taxa de asma nesse período. Há locais com altos índices de queimadas concomitantemente com altas taxas da enfermidade respiratória, como, por exemplo, Marabá Paulista e Santo Anastácio. Entretanto, há locais com baixo número de queimada e que, ainda assim, continuam apresentando taxas elevadas da doença. Constatamos uma baixa associação $(r=-0,02 ; p$-valor $=0,888)$ entre a ocorrência da doença e o número de queimadas nessa região. Embora estudos (CANÇADO et al., 2006; HUANG et al., 2019; RAMOS et al., 2019) evidenciam uma associação entre qualidade do ar e número de pacientes com quadros asmáticos, nosso estudo não mostra correlação significativa (Tabela 1) entre taxa de asma e focos de queimada, isso tanto ao se considerar a região geográfica do município como um todo ou somente a área urbana, onde há maior concentração populacional, independentemente do ano analisado no período de 11 anos. 
Uma pesquisa que avalia o impacto da queimada da cana-de-açúcar sobre as internações por enfermidades respiratórias no município de Presidente Prudente-SP entre 2011 e 2012, também, não demonstra aumento significativo entre internações por asma no período de queima comparado ao período de não queima da cana (RAMOS et al., 2019). Resultados similares foram encontrados em um estudo com crianças realizado em Manaus, entre 2002 e 2009, no qual não se observou significância entre internações por doenças respiratórias e focos de queimadas (FILHO et al., 2013).

Figura 5 - Distribuição espacial da taxa de asma em crianças na RRAS-11 de 2008 a 2018 por período sazonal.

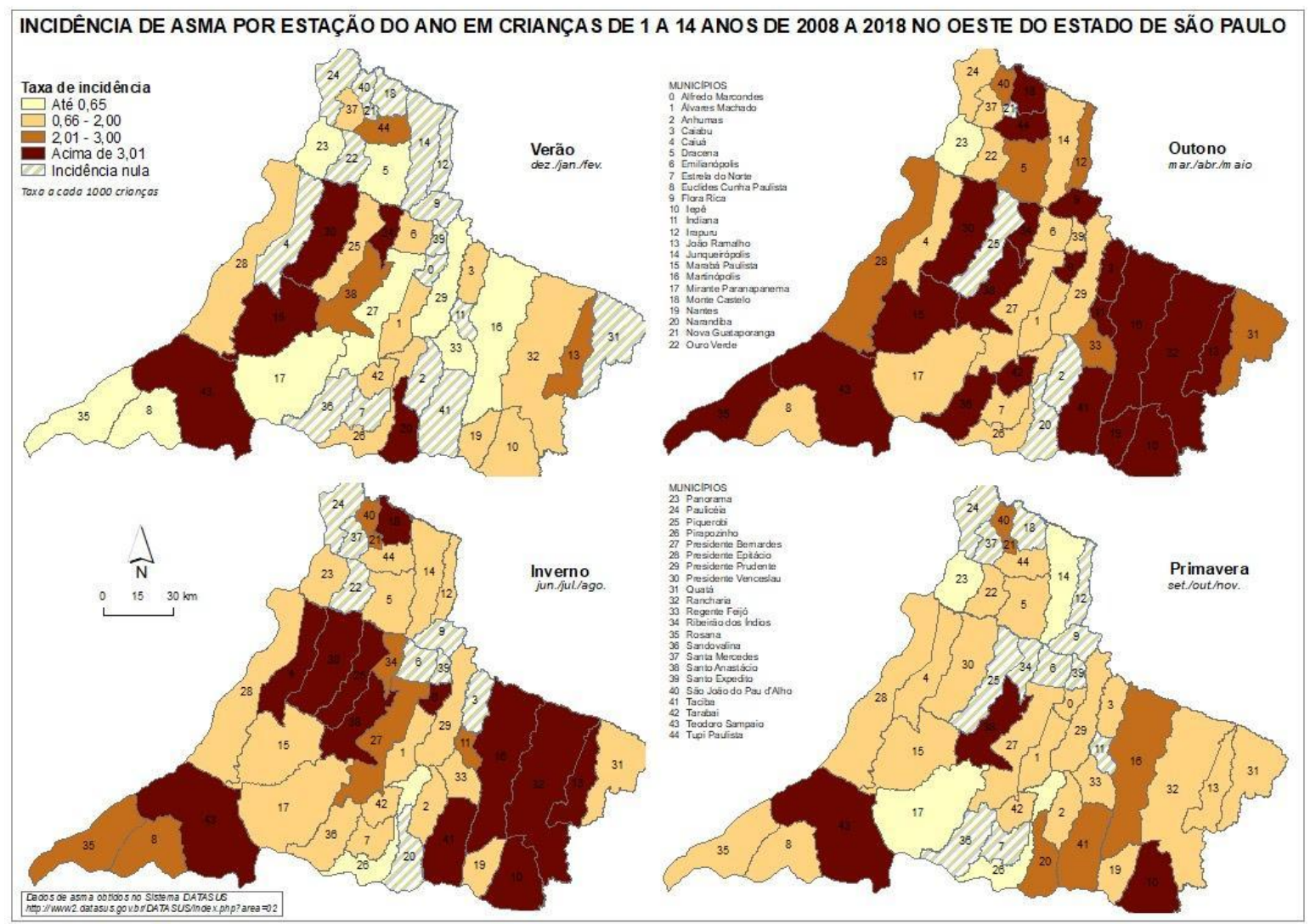

Fonte: Autores. 
Mapeamento espaço - temporal de uma década de incidência de asma em crianças em municípios Paulistas.

Cattini, M.M; Silva, R.C.R; Pimenta, M.V; Giuffrida, R; Cicerelli, R.E; Osco, L.P Junior, J.M; Carapeba, B.M.C.B.M. \& Ramos, A.P. 
Figura 6 - Demonstrativo da distribuição espacial dos focos de queimada na RRAS-11 em 2008 para o município e a incidência de asma.

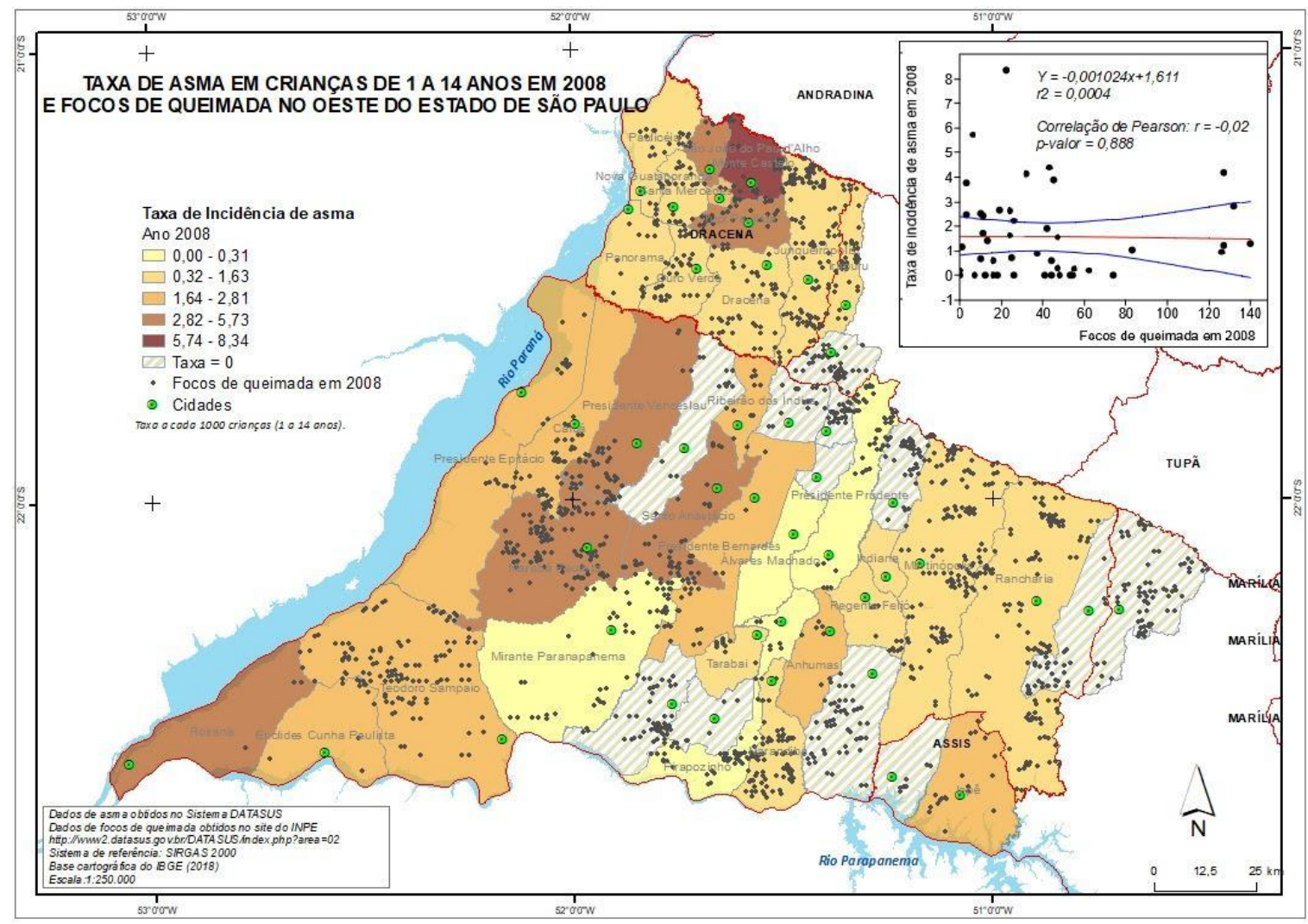

Fonte: Autores.

Tabela 1 - Correlação entre focos de queimada na RRAS-11 de 2008 a 2018 e a incidência de asma.

\begin{tabular}{c|c|c|c|c}
\hline Ano & $\begin{array}{c}\text { Correlação } \\
\text { (município) }\end{array}$ & Teste estatístico & $\begin{array}{c}\text { Correlação } \\
\text { centro urbano) } \\
\text { buffer de 10 Km }\end{array}$ & Teste estatístico \\
\hline 2008 & $\mathrm{r}=-0,022$ & $\begin{array}{c}\text { Teste } \mathrm{t}=-0,141 \\
\mathrm{r} 2=0,0005\end{array}$ & $\begin{array}{c}\mathrm{r}=-0,241 \\
\mathrm{r} 2=0,0582\end{array}$ & $\begin{array}{c}\text { Teste } \mathrm{t}=-1,631 \\
\mathrm{p} \text {-valor }=0,1103\end{array}$ \\
\hline 2009 & $\mathrm{r}=0,189$ & $\begin{array}{c}\text { Teste } \mathrm{t}=1,262 \\
\mathrm{p} 2=0,0357\end{array}$ & $\begin{array}{c}\mathrm{r}=-0,04412 \\
\mathrm{r} 2=0,0020\end{array}$ & $\begin{array}{c}\text { Teste } \mathrm{t}=-0,290 \\
\mathrm{p} \text {-valor }=0,773\end{array}$ \\
\hline 2010 & $\mathrm{r}=0,074$ & $\begin{array}{c}\text { Teste } \mathrm{t}=0,487 \\
\mathrm{p} \text {-valor }=0,629\end{array}$ & $\begin{array}{c}\mathrm{r}=-0,133 \\
\mathrm{r} 2=0,0178\end{array}$ & $\begin{array}{l}\text { Teste } \mathrm{t}=-0,882 \\
\mathrm{p} \text {-valor }=0,382\end{array}$ \\
\hline 2011 & $\mathrm{r} 2=0,005$ & $\mathrm{r}=0,091$ & Teste $\mathrm{t}=0,601$ \\
\hline
\end{tabular}




\begin{tabular}{|c|c|c|c|c|}
\hline & $\mathrm{r} 2=0,001$ & $\mathrm{p}$-valor $=0,843$ & $\mathrm{r} 2=0,0083$ & $\mathrm{p}$-valor $=0,551$ \\
\hline 2012 & $\begin{aligned} r & =0,046 \\
r 2 & =0,0021\end{aligned}$ & $\begin{array}{c}\text { Teste } \mathrm{t}=0,300 \\
\mathrm{p} \text {-valor }=0,766\end{array}$ & $\begin{array}{c}r=-0,124 \\
r 2=0,0153\end{array}$ & $\begin{array}{l}\text { Teste } t=-0,818 \\
p \text {-valor }=0,418\end{array}$ \\
\hline 2013 & $\begin{aligned} r & =0,244 \\
r 2 & =0,0597\end{aligned}$ & $\begin{array}{l}\text { Teste } \mathrm{t}=1,652 \\
\mathrm{p} \text {-valor }=0,106\end{array}$ & $\begin{aligned} r & =0,282 \\
r 2 & =0,0796\end{aligned}$ & $\begin{array}{l}\text { Teste } t=1,929 \\
p \text {-valor }=0,060\end{array}$ \\
\hline 2014 & $\begin{aligned} r & =0,030 \\
r 2 & =0,0009\end{aligned}$ & $\begin{array}{l}\text { Teste } \mathrm{t}=0,197 \\
\mathrm{p} \text {-valor }=0,844\end{array}$ & $\begin{array}{c}r=-0,132 \\
r 2=0,0176\end{array}$ & $\begin{array}{l}\text { Teste } t=-0,877 \\
p \text {-valor }=0,386\end{array}$ \\
\hline 2015 & $\begin{aligned} r & =0,047 \\
r 2 & =0,0022\end{aligned}$ & $\begin{array}{l}\text { Teste } \mathrm{t}=0,309 \\
\mathrm{p} \text {-valor }=0,759\end{array}$ & $\begin{array}{c}r=-0,032 \\
r 2=0,0010\end{array}$ & $\begin{array}{l}\text { Teste } t=-0,210 \\
p \text {-valor }=0,835\end{array}$ \\
\hline 2016 & $\begin{array}{c}r=-0,140 \\
r 2=0,0197\end{array}$ & $\begin{array}{l}\text { Teste } \mathrm{t}=-0,929 \\
\mathrm{p} \text {-valor }=0,358\end{array}$ & $\begin{array}{c}r=-0,132 \\
r 2=0,0174\end{array}$ & $\begin{array}{l}\text { Teste } t=-0,874 \\
p \text {-valor }=0,387\end{array}$ \\
\hline 2017 & $\begin{aligned} r & =0,060 \\
r 2 & =0,0036\end{aligned}$ & $\begin{array}{c}\text { Teste } \mathrm{t}=0,397 \\
\mathrm{p} \text {-valor }=0,693\end{array}$ & $\begin{array}{c}r=-0,025 \\
r 2=0,0006\end{array}$ & $\begin{array}{l}\text { Teste } t=-0,166 \\
p \text {-valor }=0,867\end{array}$ \\
\hline 2018 & $\begin{array}{c}r=-0,031 \\
r 2=0,0009\end{array}$ & $\begin{array}{l}\text { Teste } \mathrm{t}=-0,204 \\
\mathrm{p} \text {-valor }=0,839\end{array}$ & $\begin{array}{c}r=-0,127 \\
\mathrm{r} 2=0,0162\end{array}$ & $\begin{array}{l}\text { Teste } t=-0,841 \\
p \text {-valor }=0,405\end{array}$ \\
\hline $\begin{array}{c}\text { Todo o } \\
\text { período } 2008 \text { a } \\
2018\end{array}$ & $\begin{aligned} r & =0,174 \\
r 2 & =0,0301\end{aligned}$ & $\begin{array}{c}\text { Teste } \mathrm{t}=1,157 \\
\mathrm{p} \text {-valor }=0,254\end{array}$ & $\begin{array}{c}r=-0,141 \\
r 2=0,0200\end{array}$ & $\begin{array}{l}\text { Teste } t=-0,937 \\
p \text {-valor }=0,354\end{array}$ \\
\hline
\end{tabular}

A asma é uma doença que deve ser notificada pelos profissionais de saúde com o intuito de alimentar o banco de dados epidemiológico para o mapeamento e criação de políticas direcionadas às áreas de maior impacto. O presente estudo utilizou tal base (DATASUS) para o levantamento de informações e posterior análise. Sendo assim, é preciso considerar as possíveis subnotificações, diagnósticos incorretos e preenchimento incompleto de prontuários médicos, fatores estes que deixam de fazer parte da estatística e criam defasagem no sistema. Isso pode implicar em consequências que produzem limitações em informações sobre a saúde nacional, além de dificultar a criação de ferramentas que podem ilustrar as variadas regiões do país. Ainda, como no estudo de Ramos et al. (2019), outras variáveis poderiam ser testadas tais como metano, dióxido de carbono ( $\mathrm{CO} 2)$, nitrogênio ( $\mathrm{NO} 2)$ e ozônio $(\mathrm{O} 3)$, porém a falta de dados atmosféricos e meteorológicos na região prejudica a obtenção de resultados concludentes. Contudo, apesar da deficiência e limitação, há possibilidade de obtenção de dados e formação de conceitos que podem ajudar consideravelmente o sistema de saúde nacional. 


\section{CONSIDERAÇÕES FINAIS}

Este trabalho apresenta a contribuição original sobre a caracterização da distribuição espaço-temporal da incidência de asma em crianças de 1 a 14 anos nos 45 municípios da $11^{\mathrm{a}}$ Rede Regional de Atenção à Saúde do estado de São Paulo. Constatou-se que a ocorrência dessa doença nos municípios não apresenta dependência espacial, porém existe um acometimento maior de casos em determinados municípios em comparação a outros, caracterizando uma distribuição heterogênea dessa enfermidade na RRAS 11. Verificamos uma associação entre a taxa de incidência de asma e a sazonalidade climática, identificando maior incidência em estações do ano com temperatura mais baixa (inverno, por exemplo). Não obstante, a região em estudo apresenta uma tendência à queda substancial nos casos de asma em crianças no período de 2008 a 2018, o que corrobora com os achados sobre a doença em outras localidades do Brasil. Ademais, o acordo entre as usinas e o governo do Estado de São Paulo para o fim da queima da palha da cana-de-açúcar, a partir de janeiro de 2018, pode explicar a continuidade da redução das taxas dessa doença na região. Grande parte dos fatores descritos podem estar em associação às medidas de saúde pública implementadas nos últimos anos para o combate à doença. Os resultados deste trabalho contribuem para direcionar futuras ações de medidas preventivas e sociais na RRAS 11, uma vez que não se tem notícias sobre um trabalho dessa natureza até então nessa que é a maior região do estado de SP, com mais de 1 milhão de habitantes. Tais achados, contribuem, principalmente para apoiar ações nas regiões que demonstraram maior avanço da asma nos últimos 10 anos (2008 a 2018) e que necessitam dessa maneira, de maiores intervenções governamentais. Concluímos que essa pesquisa contribui para uma análise epidemiológica e posterior criação de modelos assistenciais necessários, de acordo com a demanda local a ser estudada. Como trabalhos futuros recomendamos que as taxas de asma por município sejam correlacionadas com outras variáveis ambientais, como o uso e ocupação do solo, pluviosidade da região, direção dos ventos. Sugerimos, ainda, verificar se houve focos de queimadas em municípios vizinhos às cidades que apresentaram altas taxas da doença, devido, por exemplo, à direção dos ventos nessa região direcionar a fumaça para tais cidades.

\section{AGRADECIMENTOS}

Os autores agradecem ao CNPq pela bolsa PIBIC/UNOESTE 2019-2020 concedida ao primeiro autor para a realização da pesquisa.

\section{REFERÊNCIAS}

AMÂNCIO, C. T.; NASCIMENTO, L. F. C. Asma e poluentes ambientais: um estudo de séries temporais. Rev Assoc Med Bras, v. 58(3), p.302-307, 2012.

AMARAL LM; PALMA PV; LEITE ICG. Evolução das políticas públicas e programas de controle da asma no Brasil sob a perspectiva dos consensos. J Bras Pneumol, v. 38(4), p.518-525, 2012. 
ANSELIN, L. Local indicators of spatial association - LISA. Geographical Analysis v.27, p.91-115, 1995.

BARNETT, A. G., WILliAMS, G. M., SCHWARTZ, J., NELLER, A. H., BEST, T. L., PETROESCHEVSKY, A. L., et al. Air pollution and child respiratory health: a case-crossover study in Australia and New Zealand. Am J Respir Crit Care Med, v. 171(11), 2005.

BEASLEY, R. Worldwide variation in prevalence of symptoms of asthma, allergic rhinoconjunctivitis, and atopic eczema: ISAAC. The International Study of Asthma and Allergies in Childhood (ISAAC) Steering Committee. Lancet, v. 351. p. $1225-1232$. 1998.

Disponível em https://middleeast.thelancet.com/journals/lancet/article/PIIS0140-6736(97)07302-9/references>

BOIN, M. N. Chuvas e Erosões no Oeste Paulista: uma análise climatológica aplicada. Tese de Doutorado. IGCE / UNESP de Rio Claro - SP, 2000. 264p.

BRAZ, R. M., GUIMARÃES, R. F., CARVALHO JÚNIOR, O. A. de, \& TAUIL, P. L. (2014). Spatial dependence of malaria epidemics in municipalities of the Brazilian Amazon. Revista Brasileira de Epidemiologia, 17(3), 615-628. doi:10.1590/1809-4503201400030004

CAMPOS, H. S. Asma grave. JBM, v. 103(2), 2015. FIOCRUZ, MS. Disponível em: < http://files.bvs.br/upload/S/0047-2077/2016/v103n2/a5400.pdf> [Acessado em 24/10/2017].

CANÇADO J.E.D.; SALDIVA P.H.N.; PEREIRA L.A.A.; LARA L.B.L.S.; ARTAXO P.; MARTINELLI L.A.; ARBEX M.A.; ZANOBETTI A.; BRAGA A.L.F. The Impact of Sugar Cane-Burning Emissions on the Respiratory System of Children and the Elderly. Environmental Health Perspectives, v. 114(5), 2006.

CARDOSO, T. A.; RONCADA, C.; SILVA, E. R.; PINTO, L. A.; JONES, M. H.; STEIN, R. T.; PITREZ, P. M. Impacto da asma no Brasil: análise longitudinal de dados extraídos de um banco de dados governamental brasileiro. Revista brasileira de epdemiologia, v. 43 (3), p. 163-168. 2017.

CARDOSO, P. V.; SEABRA, V.S.; BASTOS, I.B.; COSTA, E.C.P. A importância da análise espacial para tomada de decisão: um olhar sobre a pandemia de covid-19. Tamoios, v. 16 (1), p. 125-137. 2020. https://doi.org/10.12957/tamoios.2020.50440

CHAVES LE; NASCIMENTO LFC; RIZOL PMSR. Modelo fuzzy para estimar o número de internações por asma e pneumonia sob os efeitos da poluição do ar. Rev Saude Publica, v. 51(55), 2017. https://doi.org/10.1590/S1518-8787.2017051006501.

COSTA E; CAETANO R; WERNECK GL; BREGMAN M; ARAÚJO DV; RUFINO R. Estimativa de custo da asma em tratamento ambulatorial: estudo com dados de mundo real. Rev Saude Publica, v. 52(27), 2018. https://doi.org/10.11606/S1518-8787.2018052000153.

DATASUS. Departamento de Informática do Sistema Único de Saúde do Brasil. Disponível em: http://tabnet.datasus.gov.br/.

DENT, B. D.; TORGUSON, J.; HODLER, T. Cartography: Thematic Map Design. $6^{\text {th }}$ ed. McGraw-Hill, Georgia, 368p. 2009.

DO VALE, V.H.C.; LEAL, T.S.L; MELLO, L.O.F.; BRAZ. R.M.S. As geotecnologias na identificação de fatores influenciadores da vulnerabilidade da disseminação da covid-19: um estudo de caso na fase de reabertura do município de Niterói. Tamoios, v. 16(3), p. 4-15, 2020. https://doi.org/10.12957/tamoios.2020.55746.

EDER, W., EGE, M. J., VON MUTIUS, E. The asthma epidemic. N Engl J Med, v. 355(21), p. 2226-35, 2006. https://doi.org/10.1056/NEJMra054308.

EZEQUIEL OS.; GAZETA GS.; FREIRE NMS. Prevalência dos atendimentos por crise de asma nos serviços públicos do município de Juíz de Fora (MG). J Bras Pneumol, v. 33(1), p.20-27, 2007.

FELIZOLLA, MLBM., VIEGAS CAA, ALMEIDA M, FERREIRA F., SANTOS MCA. Prevalência de asma brônquica e de sintomas a ela relacionados em escolares do Distrito Federal e sua relação com o nível socioeconômico. J Bras Pneumol, v. 31(6), p. 486-91, 2005. 
FERNANDES, S. S. C.; SOLÉ, D.; CAMARGOS, P.; ANDRADE, C. R.; IBIAPINA, C. C. Fatores associados à expressão da asma em adolescentes. Jornal Brasileiro de Pneumologia, v. 44 (1), p. 12-17. 2018.

FILHO V.S.A.; ARTAXO P.; HACON S.; CARMO C.N.; CIRINO G. Aerossóis de queimadas e doenças respiratórias em crianças, Manaus, Brasil. Rev Saúde Pública, v.47(2), p. 239-47. 2013. DOI: 10.1590/S0034-8910.2013047004011.

FRANCO, J. M.; GURGEL, Q. R.; SOLÉ, D. Epidemiologia da Asma. Revista Brasileira de Alergia Imunopatológica. v. 29 (4), 2006.

GINA - Global Initiative for Asthma. Global Strategy for Asthma Management and Prevention, 2016. Disponível em < https://ginasthma.org/wp-content/uploads/2016/04/wms-GINA-2016-main-report-final.pdf>. Acesso em 2019.

HUANG R.; HU Y.; RUSSELL A.G.; MULHOLLAND J.A.; ODMAN M.T. The Impacts of Prescribed Fire on PM2.5 Air Quality and Human Health: Application to Asthma-Related Emergency Room Visits in Georgia, USA. Int. J. Environ. Res Public Health, v. 16(2312), 2019. doi:10.3390/ijerph16132312.

IBGE. Instituto Brasileiro de Geografia e Estatística. Características da população no Brasil. 2010. Disponível em: https://www.ibge.gov.br/.

INPE. Instituto Nacional de Pesquisas Espaciais. Disponível em: http:/queimadas.dgi.inpe.br/queimadas/bdqueimadas.

KRYGIER, J.; WOOD, D. Making Maps: A visual guide to map design for GIS. New York: Guilford Publications. 2005. $303 p$.

LAI C.K; BEASLEY R.; CRANE J.; FOLIAKI S.; SHAH J.; WEILAND S. Global variation in the prevalence and severity of asthma symptoms: phase three of the International Study of Asthma and Allergies in Childhood (ISAAC). Thorax, v. 64(6), p.476-83, 2009. https://doi.org/10.1136/ thx.2008.106609.

MANNINO, D. M., HOMA, D. M., REDD, S. C. Involuntary smoking and asthma severity in children: data from the Third National Health and Nutrition Examination Survey. Chest. v. 122 (2), 2002.

SOUZA J.M.; GIUFFRIDA, R.; RAMOS, A.P.M.; MORCELI, G.; COELHO, C.H.; RODRIGUES, M. V. P. Mother-to-child transmission and gestational syphilis: Spatial-temporal epidemiology and demographics in a Brazilian region. PLOS Neglected Tropical Diseases, v.13 (2), 2019. https://doi.org/10.1371/journal.pntd.0007122.

MARQUES GA; WENDT A; WEHRMEISTER FC. Evolução temporal e fatores associados a asma e sibilância em escolares no Brasil. Bras Pneumol, v. 45(3), 2019. http://dx.doi.org/10.1590/1806-3713/e20180138.

MONTEIRO, C. A. de F. Geossistemas: a história de uma procura. São Paulo: Contexto, 2000.

NETO, H. J. C.; ROSÁRIO, N.A; SOLÉ, D.; Latin American ISAAC Group. Asthma and Rhinitis in South America: How Different They are From Other Parts of the World. Allergy Asthma Immunol Res. v.4 (2), p.62-67, 2012. https://doi.org/10.4168/aair.2012.4.2.62.

PATElla V.; FlORIO G.; MAGLIACANE D.; GIULIANO A.; CRIVELlARO M.G.; DI BARTOLOMEO D.; GENOVESE A.; PALMIERI M.; POSTIGLIONE A.; RIDOLO E.; SCALETTI C.; VENTURA M.T.; ZOLLO A.; Air pollution and climate change task force of the italian society of allergology, asthma and clinical immunology (siaaic). Urban air pollution and climate change: "The Decalogue: Allergy Safe Tree" for allergic and respiratory diseases care. Clin Mol Allergy, v. 16(20), 2018. https://doi.org/10.1186/s12948-018-0098-3.

RAMOS, A. P. M.; MARCATO JUNIOR, J. ; DECANINI, M. M. S. ; PUGLIESI, E. A.; OLIVEIRA, R. F.; PARANHOS FILHO, A. C. Avaliação qualitativa e quantitativa de métodos de classificação de dados para o mapeamento coroplético. Rbc. Revista Brasileira de Cartografia (online), v. 68, p. 609-629, 2016.

RAMOS D.; PESTANA P.R.S.; TREVISAN I.B.; CHRISTOFARO D.G.D.; TACAO G.Y.; CORIPIO I.C.; FERREIRA A.D.; RAMOS E.M.C. Impacto da queima da cana-de-açúcar sobre internações hospitalares por doenças respiratórias. Ciência \& Saúde Coletiva, v. 24(11), p.4133-4140, 2019. DOI: 10.1590/1413-812320182411.32402017

REN H, SHANG Y, ZHANG S (2020) Measuring the spatiotemporal variations of vegetation net primary productivity in Inner Mongolia using spatial autocorrelation. Ecol Indic 112:106108. https://doi.org/10.1016/j.ecolind.2020.106108 
RODRIGUES A.M.; PITREZ P.M. Asma de difícil controle na criança: um panorama para o pediatra. Boletim Científico de Pediatria, v.1(1), 2012.

RODRIGUES C.G.; VORMITTAG E.M.P.A.; CAVALCANTE J.A.; SALDIVA P.H.N. Projeção da mortalidade e internações hospitalares na rede pública de saúde atribuíveis à poluição atmosférica no Estado de São Paulo entre 2012 e 2030. R. bras. Est. Pop, v.32 (3), p.489-509, 2015. DOI http://dx.doi.org/10.1590/S0102-3098201500000029.

RODRIGUES, P. C. O.; IGNOTTI, E.; ROSA, A. M.; HACON, S. S Distribuição espacial das internações por asma em idosos na Amazônia Brasileira. Revista brasileira de epidemiologia, v. 13(3), p. 523-532, 2010.

RONCADA, C.; CARDoso, T. A.; BUGAnÇA, B. M.; BISChOFF, L. C.; SOldERA, K.; PITREZ, P. M. Níveis de conhecimento sobre asma de pais de crianças asmáticas. Einstein (São Paulo), v. 16(2), p. 1-6, 2018.

SEEG. Sistema de Estimativas de Emissões e Remoções de Gases de Efeito Estufa. Disponível em: http://plataforma.seeg.eco.br/map?cities=true.

SILVA, E. C. F.; DIAS, G. A. C. The pathogenesis of asthma. Revista Hospital Universitário Pedro Ernesto, v.12 (2), 2013.

SILVA, P. R. S.; ROSA, A. M.; HACON, S. S.; IGNOTTI, E. Hospitalização de crianças por asma na Amazônia brasileira: tendência e distribuição espacial. Journal de Pediatria, v. 85 (5), p. 541-546, 2009.

SILVA, R.C.R.; BARRETO M.L.; RAMOS D.; CRUZ A.A.; CAMPOS M.O.; MALTA D.C. Tendência da asma na adolescência no Brasil: resultados da Pesquisa Nacional de Saúde do Escolar (PeNSE) 2012 e 2015. Rev Bras Epidemiol, v. 21(1), 2018. DOI: 10.1590/1980-549720180017.supl.1.

SOUZA, K. V.; BARATA, M. M. L.; SANTOS, J.; P.; C.; STRAUCH, J. C. M.; CASTRO, H. A. Analysis of the spatial distribution of the burning sugar cane and prevalence of asthma symptoms in students from Campos of the Goytacazes - RJ, period 2012 - 2013. Hygeia: Revista Brasileira de Geografia Médica e da Saúde, v. 13(24), p. 41-53, 2017.

THOMPSON ES, SAVEYN P, DECLERCQ M, MEERT J, GUIDA V, EADS CD, ROBLES ESJ, BRITTON MM (2018) Characterisation of heterogeneity and spatial autocorrelation in phase separating mixtures using Moran's I. J Colloid Interface Sci 513:180-187. https://doi.org/10.1016/j.jcis.2017.10.115

VALENÇA, L. M.; RESTIVO, P. C.; NUNES, M. S. Variação sazonal nos atendimentos de emergência por asma em Gama, Distrito Federal. Jornal Brasileiro de Pneumologia, v. 32(4), p. 284-289, 2006.

VIOTO, C. Z.; RAMOS, A. P. M.; SILVA, R. C. R.; RODRIGUES, M. V. P.; OSCO, L. P. Avaliação da distribuição espaço-temporal da asma na região do Pontal do Paranapanema entre 2008 e 2015. Revista Colloquium Vitae, v. 9, p. 33-38. 2017. DOI: $10.5747 / \mathrm{cv} .2017 . v 09$. nesp.000296

WEHRMEISTER, F. C.; MENEZES, A. M. B.; CASCAES, A. M.; MARTÍNEZ-MESA, J.; BARROS, A. J. D. Tendência temporal de asma em crianças e adolescentes no Brasil no período de 1998 a 2008. Revista de Saúde Pública, v. 46(2), p. 242-249, 2012.

ZAR H; FERKOL TW. The Global Burden of Respiratory Disease - Impact on Child Health. Pediatric Pulmonology, v. 49, p. 430-434, 2014.

\section{COMO CITAR ESTE TRABALHO}

Cattini, M.M; Silva, R.C.R; Pimenta, M.V Giuffrida, R Osco, L.P; Carapeba, B.M.C.B de Melo; Ramos, A.P; Cicerelli, R.E \& Junior, J.M. Mapeamento espaço - temporal de uma década de incidência de asma em crianças em municípios Paulistas. Revista Tamoios, São Gonçalo, v. 17, n. 2, p. 206-226, 2021. Disponível em: https://doi.org/10.12957/tamoios.2021.61191. Acesso em: DD MM. AAAA. 